\title{
In vivo targeting of ERG potassium channels in mice and dogs by a positron-emitting analogue of fluoroclofilium
}

\author{
Sang Wook Kim", Seung Dae Yang ${ }^{2}$, \\ Byung-Jae $\mathrm{Ahn}^{3}$, Jeong Hoon Park ${ }^{3}$, \\ Dong Soo Lee ${ }^{4}$, Guido Gessner ${ }^{5}$, \\ Stefan H. Heinemann ${ }^{5}$, Wilhelm Herdering ${ }^{6}$ \\ and Kook Hyun $\mathrm{Yu}^{3,7}$ \\ ${ }^{1}$ Radiaton Application Research Division \\ Korea Atomic Energy Research Institute \\ 150, Dukjin-dong, Yuseong \\ Daejeon 305-353, Korea \\ ${ }^{2}$ Laboratory of Accelerator Development \\ Korea Institute of Radiological \& Medical Sciences \\ 215-4 Gongleung-dong, Nowon-gu \\ Seoul 139-706, Korea \\ ${ }^{3}$ Department of Chemistry, Dongguk University \\ 3 Pil-dong, Chung-gu \\ Seoul 100-715, Korea \\ ${ }^{4}$ Department of Nuclear Medicine \\ Seoul National University College of Medicine \\ 28 Yeongun-dong, Chongro-gu \\ Seoul 110-744, Korea \\ ${ }^{5}$ Research Unit Molecular and Cellular Biophysics \\ Medical Faculty of the Friedrich Schiller University Jena \\ Drackendorfer Str. 1, D-07747 Jena, Germany \\ ${ }^{6}$ Institut für Anorganische und Angewandte Chemie \\ Universität Hamburg, Martin-Luther-King-Platz 6 \\ D-20146 Hamburg, Germany \\ ${ }^{7}$ Corresponding author: Tel, 82-2-2260-3709; \\ Fax, 82-2-2264-4201; E-mail, yukook@dongguk.edu
}

\section{Accepted 16 June 2005}

Abbreviations: cERG, canine ether a go-go related gene; hEAG, human ether a go-go; hERG, human ERG; PET, positron emission tomography

\footnotetext{
Abstract

The antiarrhythmic clofilium is an efficient blocker of hERG1 potassium channels that are strongly expressed in the heart. Therefore, derivatives of clofilium that emit positrons might be useful tools for monitoring hERG1 channels in vivo. Fluoroclofilium (F-clofilium) was synthesized and its channel-blocking properties were determined for hERG1 and hEAG1 channels expressed in HEK 293 cells and in Xenopus oocytes. When applied
}

extracellularly in the whole-cell patch-clamp con figuration, F-cloflium exhibited a slower onset of block when compared with clofilium, presumably owing to its lower membrane permeability. When applied in the inside-out configuration at the intracellular membrane side, it blocked hEAG1 channels almost as efficiently as clofilium (IC $_{50}$ $1.37 \mathrm{nM}$ and $0.83 \mathrm{nM}$, respectively). Similar results were obtained for hERG1, showing Fclofilium is a potent hERG1 and hEAG1 channel blocker once it has reached the intracellularly accessible target site at the channel. Using the ${ }^{18} \mathrm{~F}$ labeled analog we studied the in vivo binding and distribution of F-clofilium in mice and a dog. Greatest activity was found in kidneys and bones. A small but significant enrichment of activity in the dog myocardium known for its expression of cERG1 channels allowed to depict the myocardium of a living dog by PET. Thus, F-clofilium is a useful tool for imaging hERG channels in living organisms.

Keywords: anti-arrhythmia agent; clofilium; dog; ERG1 potassium channel; heart; positron-emission tomography

\section{Introduction}

The quaternary ammonium derivative clofilium is well known for its class III antiarrhythmic properties (Borzak et al., 1997). hERG $\mathrm{K}^{+}$channels are blocked by clofilium in a nanomolar range and this binding is believed to be the reason for its antiarrhythmic action (Suessbrich et al., 1997). However, block of hERG1 $\mathrm{K}^{+}$channels in the ventricles can also cause a prolongation of the QT-interval ultimately leading to fatal torsades de pointes (Vandenberg et al., 2001).

Recent studies revealed also that hERG $\mathrm{K}^{+}$channels are abundantly expressed in a variety of tumor cells. hERG conductance markedly promotes $\mathrm{H}_{2} \mathrm{O}_{2-}$ induced apoptosis of various tumor cells, whereas hERG expression facilitates the tumor cell proliferation caused by tumor necrosis factor (TNF) ligand (TNF-alpha) (Wang et al., 2002). Similarly, hEAG1 channels that are closely related to $\mathrm{hERG}$ are also found in tumor cell lines and are believed to play a role in malignant cell growth (Pardo et al., 1999; Gavrilova-Ruch et al., 2002). Thus, specific markers for hERG1 and hEAG1 channels could find appli- 
cation in both cardiovascular and oncological research.

${ }^{14} \mathrm{C}$-labeled clofilium showed high myocardial uptake (Su et al., 1984) and radioactive labeling of its aromatic residue by several iodine isotopes has been carried out (Molly et al., 1983). Recently we synthesized fluoroclofilium (F-clofilium, Figure 1A) (Yu et al., 2003) and its ${ }^{18} \mathrm{~F}$-labelled analog $\left({ }^{18} \mathrm{~F}\right.$-clofilium) as a potential cardiac imaging agent for studies by PET (Positron Emission Tomography), a powerful method to measure specific binding of ligands and metabolism in living species including humans (Hatazawa et al., 1991; Schweiger et al., 1991).

Often substitution of a hydrogen atom by fluorine does not disturb much (Reivich et al., 1985) the binding properties to a binding site but a disturbance by the fluorine cannot be excluded. Also the metabolic fate can be affected (Degrado et al., 1992). Therefore at first we investigated the binding properties of F-clofilium to hERG1 and hEAG1 channels in vitro. Subsequently we studied the biodistribution of radioactive ${ }^{18} \mathrm{~F}$-clofilium in mice and dogs.

\section{Materials and Methods}

\section{Synthesis of F-clofilium and ${ }^{18} \mathrm{~F}$-clofilium}

Synthesis of F-clofilium and ${ }^{18} \mathrm{~F}$-clofilium was carried out as described earlier (Yu et al., 2003).

\section{Distribution coefficients for clofilium and F-clofilium in the biphasic system 1-octanol/water}

Three milligrams of clofilium (or F-clofilium) were dissolved in a mixture of $5 \mathrm{ml}$ of 1 -octanol and $5 \mathrm{ml}$ of water. The mixture was vortexed for $1 \mathrm{~min}$ and was allowed to separate for $4 \mathrm{~h}$ into two phases. The ammonium compound content of the organic and aqueous phase was measured by HPLC (50 $\mu$; Nova-Pak C-18, $3.9 \times 300 \mathrm{~mm} ; 277 \mathrm{~nm}$ ). As eluent for measurements of the organic phase $\mathrm{MeOH} / 0.05$ $\mathrm{M} \mathrm{K}_{2} \mathrm{HPO}_{4}$ with $10 \% \mathrm{MeOH}$ in a ratio of $60: 40$ was used. The aqueous phase was analyzed by the eluents $\mathrm{MeOH} / 0.05 \mathrm{M} \mathrm{K}_{2} \mathrm{HPO}_{4}$ with $10 \% \mathrm{MeOH}$ in a ratio of 90:10. The content of clofilium (or fluoroclofilium) in each phase was 10 times. Calibration of the HPLC system was carried out for clofilium and fluoroclofilium in each of the two applied eluent systems. The mean distribution coefficient in the system 1-octanol/water was $4.3 \pm 0.3$ for clofilium and $2.6 \pm$ 0.5 for fluoroclofilium.

\section{Expression of hERG1 in mammalian cell lines}

hERG1 channels were stably expressed in HEK 293 cells (Schönherr et al., 1999). Cells were cultured in
Dulbecco's modified Eagle's (Invitrogen) / F12 medium (ratio 1:1) supplemented with $10 \%$ FCS. The cell line was maintained at $37^{\circ} \mathrm{C}$ in a humidified atmosphere with $5 \% \quad \mathrm{CO}_{2}$ and subcultured every 3-4 days. All cells were used within passage numbers 3 to 25. For electrophysiological recordings cells were plated on 35-mm petri-dishes.

\section{Expression of hERG1 and hEAG1 in Xenopus oocytes}

For heterologous expression in Xenopus oocytes capped mRNA of hERG1 (Schönherr et al., 1996) and hEAG1 (Schönherr et al., 2000) subcloned in pGEM$\mathrm{HE}$ was synthesized in vitro with T7 RNA polymerase (mMESSAGE mMACHINE kit, Ambion, Austin, TX). Oocyte preparation and injection of RNA were performed as described previously (Schönherr et al., 1999).

\section{Electrophysiological recordings}

As patch-clamp amplifier we used an EPC 9 (HEKA Elektronik, Lambrecht, Germany). For recordings from mammalian cells the patch pipettes were fabricated from borosilicate glass (Kimble Glass, Vineland, NJ) with resistances of 1-2 M $\Omega$. Unless stated otherwise, the pipettes were filled with (in $\mathrm{mM}$ ): $130 \mathrm{KCl}$, $2.56 \mathrm{MgCl}_{2}, 10$ EGTA, 10 HEPES, pH 7.4 (adjusted with $\mathrm{KOH}$ ). The standard bath solution contained (in $\mathrm{mM}$ ): $110 \mathrm{NaCl}, 40 \mathrm{KCl}, 2 \mathrm{MgCl}_{2}, 2 \mathrm{CaCl}_{2}, 10 \mathrm{HEPES}$, $\mathrm{pH} 7.4(\mathrm{NaOH})$. Currents were measured in the whole-cell configuration with access resistances below $3 \mathrm{M} \Omega$. Series resistance compensation was applied to $80-90 \%$.

For macro-patch recordings from Xenopus oocytes in the inside-out configuration the patch pipettes were formed from aluminum silicate glass (Hilgenberg, Malsfeld, Germany) with tip resistances of $12 \mathrm{M} \Omega$. Standard external solution was composed of (in $\mathrm{mM}$ ): 103.6 NaAsp, $11.4 \mathrm{KCl}, 1.8 \mathrm{CaCl}_{2}, 10$ HEPES, pH 7.4 $(\mathrm{NaOH})$. Standard internal solution contained (in $\mathrm{mM}$ ): $120 \mathrm{KAsp}, 15 \mathrm{KCl}, 10 \mathrm{EGTA}, 10$ HEPES, pH $7.4(\mathrm{KOH})$. Pipette tips for both types of experiments were firepolished immediately before use. All experiments were performed at room temperature $\left(20-23^{\circ} \mathrm{C}\right)$.

All chemicals used were of high grade, obtained from Sigma (Taufkirchen, Germany).

F-clofilium was dissolved in the bath solution immediately before the experiments. It was applied to the cells via exchange of the bath solution; this was complete in about $1 \mathrm{~min}$. Blocker effects were monitored by repetitive depolarizations. Current block was analyzed by plotting the remaining current, normalized to the control current before blocker application as a function of the blocker concentration. $I C_{50}$ values 
were obtained by fitting a Hill equation to these data:

$\mathrm{I} / \mathrm{I}_{\text {control }}=1 /\left(1+\left([\right.\right.$ blocker $\left.\left.] / \mathrm{IC}_{50}\right) n_{H}\right) \ldots \ldots \ldots$ with the half-maximum inhibition concentration $I C_{50}$ and the Hill coefficient $n_{H}$. To quantify the time course of onset of block an exponential function:

$I(t)=I(0)-(I(0)-I(\infty))\left(1-\exp \left(-\left(t-t_{0}\right) / \tau_{b}\right)\right) \cdots(2)$ was employed. $I(0)$ and $I(\infty)$ are the current amplitudes before blocker application (at time $t_{0}$ ) and after block equilibration, respectively. $\tau_{b}$ is the time constant of onset of block.

Data acquisition and analysis were carried out with Pulse+PulseFit, PatchMaster, and FitMaster (HEKA) and IgorPro (WaveMetrics, Lake Oswego, OR) software. All data are presented as mean $\pm \operatorname{SEM}(n)$ with $n$ being the number of independent experiments. Statistical differences between two groups of data were performed using two-sided Student's $t$-test indicated as $P<0.05\left(^{*}\right)$ or $P<0.005\left(^{* *}\right)$.

\section{Biodistribution of radioactivity in mice after injection of ${ }^{18} \mathrm{~F}$-clofilium}

Eight Balb/c female mice (25-35 g) were injected with 1.8 $\mathrm{MBq}{ }^{18} \mathrm{~F}$-clofilium in $0.1 \mathrm{ml}$ isotonic $\mathrm{NaCl}$ solution in the tail vein. Four mice were sacrificed with diethyl ether after $15 \mathrm{~min}$, the other four after $60 \mathrm{~min}$. The organs were excised from each animal and weighed and the radioactivity was measured. The radioactive content of the blood and other organs was determined with a gamma counter. The data were decay corrected and related to activity per gram of tissue in $\%$ of injected dose (\% ID/g).

\section{PET scan}

The PET scan was carried out on an ECAT EXACT 47 scanner (Siemens Medical System, Inc., Hoffman Estates, IL/ CTI, Knoxville, TN; resolution $5 \mathrm{~mm}$ ).

A dog (25 kg, male, beagle) was anesthetized using thiopental sodium. Self-respiration was sustained and the dog laterally positioned on its right side. A transmission scan was acquired using triple ${ }^{68} \mathrm{Ge}$ sources to correct data for internal $\gamma$-absorption. After intravenous injection of $370 \mathrm{MBq}{ }^{18} \mathrm{~F}$-clofilium the radioactive compound was allowed to distribute in the animal for $30 \mathrm{~min}$. Dynamic myocardial PET data were collected during a period of $5 \mathrm{~min}$. Using the system software, two series of image slices, a coronal (horizontal) and vertical (parallel to the long axis), were acquired.

\section{Results}

\section{Block efficiency of F-clofilium}

To assay the functional activity of F-clofilium as a potent blocker of hERG potassium channels, we expressed hERG1 channels in HEK 293 cells and measured $\mathrm{K}^{+}$currents employing the whole-cell patchclamp method. Like previously shown for clofilium (Gessner et al., 2003), the onset of current block upon application of F-clofilium was very slow. When determining complete dose-response relationships from an identical cell one has to compromise precision of measurements as only about 10 min could be waited before application of the next higher concentration of blockers. Using such protocols and a holding voltage of $-100 \mathrm{mV}$, data shown in Figure $1 \mathrm{C}$ were compiled. The dose-dependent block of hERG1 current was described with a Hill equation (eq. 1) yielding an apparent $I C_{50}$ for F-clofilium of about $43 \mathrm{nM}$. In comparison, for clofilium $2.6 \mathrm{nM}$ were measured previously (Gessner et al., 2003).

This result could mean that F-clofilium blocks hERG1 channels less efficiently than clofilium. However, since clofilium has to enter the binding site in the channel cavity from the cytosol, the apparent difference could be due to different membrane permeabilities of F-clofilium and clofilium. This is quite conceivable given the extra polar group added to the alkane chain of clofilium (Figure 1A), yielding an octanol/water distribution coefficient of 2.6 compared with 4.3 for clofilium as described under Materials and Methods. Therefore, we also attempted to measure onset of block by application of either $1 \mu \mathrm{M}$ F-clofilium or clofilium (Figure 1D). The time course of current reduction was faster for clofilium (109 $\pm 12 \mathrm{~s}$ as compared to $179 \pm 31 \mathrm{~s}$ for F-clofilium), albeit for the experiments shown no statistical significance was reached. However, the time constant at $100 \mathrm{nM}$ was already about $15 \mathrm{~min}$, making it impossible to obtain faithful steady-state block data in the whole-cell configuration. Certainly, the dose-response measurements obtained for 10 min equilibration periods (Figure 1C) clearly results in an overestimation of the apparent $I C_{50}$.

In order to obtain a more direct access to the blocking properties of F-clofilium, we applied it to inside-out patches from Xenopus oocytes expressing hERG1 channels. Under inside-out conditions, hERG channels undergo a rapid rundown, which compromised the determination of accurate steady-state dose-response curves. Therefore, we applied $10 \mathrm{nM}$ of the substances to inside-out patches and observed the gradual reduction of hERG tail currents. As shown in Figure $1 \mathrm{E}$, the channels are blocked to approximately the same extent by clofilium and F-clofilium. The data shown in Figure $1 \mathrm{E}$ are compatible with a steady-state block of $82 \%$, which is equivalent to an apparent $I C_{50}$ value of $2.2 \mathrm{nM}$.

Clofilium is not only a strong blocker of hERG channels - hEAG1 channels are also inhibited by this 
A

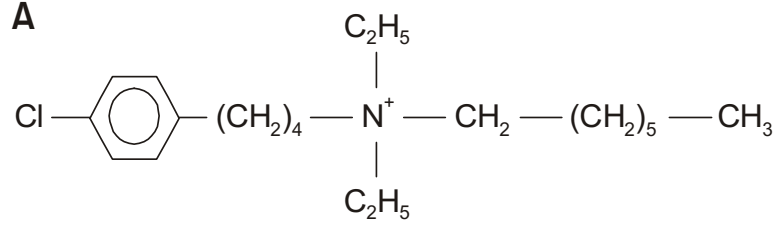

1

B
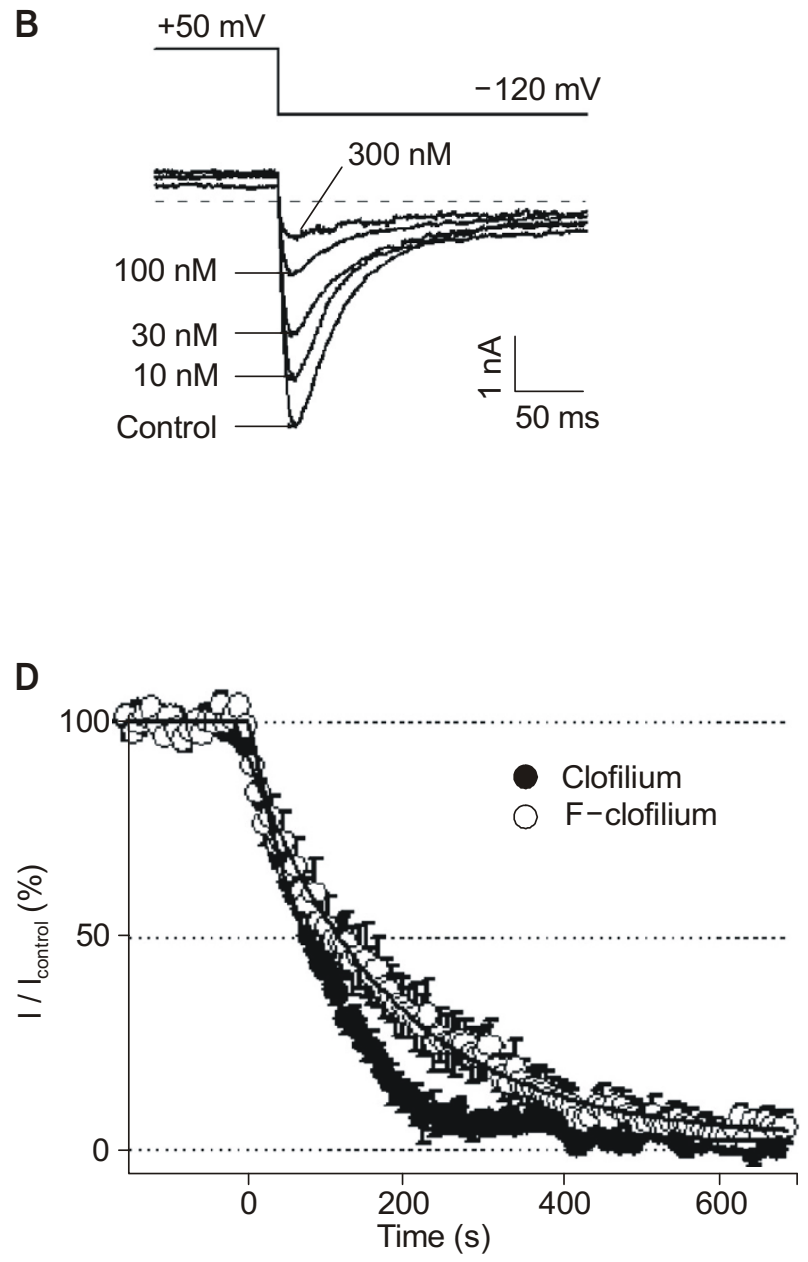<smiles>C[N+](C)(CCCCCF)CCc1ccc(Cl)cc1</smiles>

2
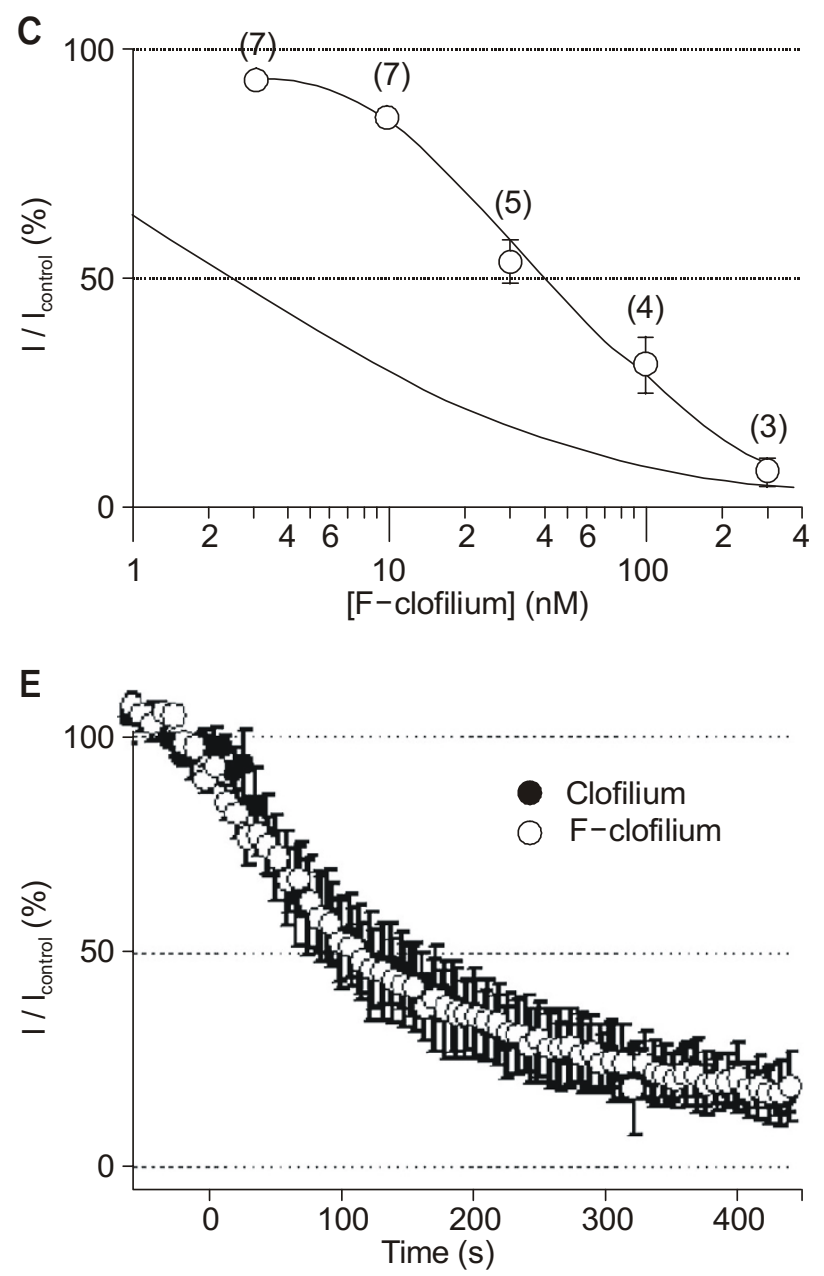

Figure 1. Inhibition of hERG1 currents by F-clofilium. A, Chemical structures of clofilium and F-clofilium. B, Superposition of whole-cell current recordings from HEK 293 cells, expressing hERG1 channels with the indicated concentrations of F-clofilium. Cells were depolarized for $1 \mathrm{~s}$ to $+50 \mathrm{mV}$ at an interval of $8 \mathrm{~s}$. Peak inward hERG currents were measured during the deactivation phase at $-120 \mathrm{mV} 10 \mathrm{~min}$ after blocker application. C, The current magnitudes, normalized to the control currents, as a function of the blocker concentration. The error bars are SEM values for the indicated number of experiments. The superimposed curve is a fit according to the Hill equation (eq. 1) yielding $I C_{50}=43.5 \pm 5.3 \mathrm{nM}$, and $n_{H}=1.07 \pm 0.08$ for F-clofilium. As a control, block by clofilium is also indicated as dashed curve (data from Gessner \& Heinemann, 2003): $I C_{50}=2.6 \mathrm{nM}$, and $n_{H}=0.62$. D, The time course of channel block during repetitive depolarizations was assayed by application of $1 \mu \mathrm{M}$ F-clofilium (open symbols) and $1 \mu \mathrm{M}$ clofilium (filled symbols), respectively. The onset of block develops approximately monoexponentially with a time constant of $179 \pm 31 \mathrm{~s}(n=5)$ for F-clofilium. The time constant for clofilium was shorter (109 $\pm 12 \mathrm{~s}, n=4)$, but not significantly different $(P=0.08)$. $E$, Time course of $h E R G 1$ channel block in inside-out patches of Xenopus oocytes upon application of $10 \mathrm{nM}$ of the indicate substances $(n=4) .450 \mathrm{~s}$ after application of the drugs the current was reduced to $18 \%$ of the control value. Membrane patches were depolarized to $+50 \mathrm{mV}$ for $1 \mathrm{~s}$ with a repetition interval of $8 \mathrm{~s}$. Currents were measured during the repolarizing phase at $-120 \mathrm{mV}$.

substance (Gessner et al., 2003). As hEAG1 channels seem to play an important role in malignant growth, we tested whether F-clofilium is a strong blocker as well. If so, ${ }^{18} \mathrm{~F}$-cofilium might also be useful for localizing small hEAG1-expressing tumors. When applied to inside-out patches of hEAG1-expressing 
A

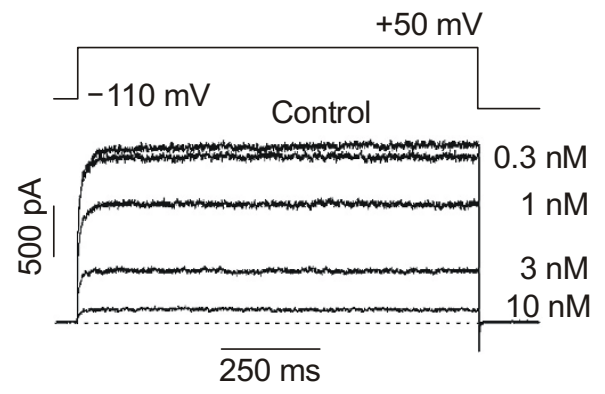

B

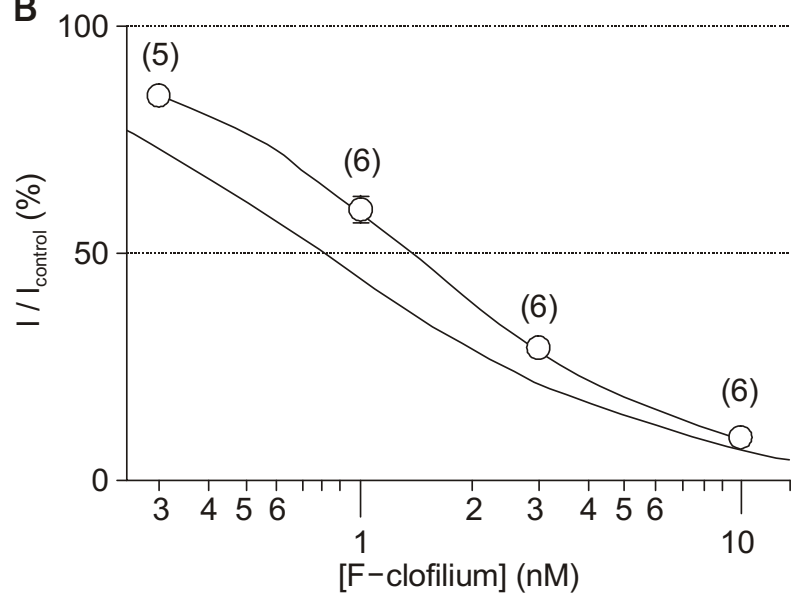

Figure 2. Inhibition of hEAG1 currents in the inside-out patch-clamp configuration by F-clofilium. A, Superposition of current recordings from membrane patches of Xenopus oocytes expressing hEAG1 channels with the indicated concentrations of F-clofilium. Cells were depolarized for $1 \mathrm{~s}$ to $+50 \mathrm{mV}$ at an interval of $7 \mathrm{~s}$. Holding potential was $-110 \mathrm{mV}$ and mean outward hEAG current was measured during the second half of the depolarization period. B, The current magnitudes, normalized to the control currents, as a function of the blocker concentration. The error bars are SEM values for the indicated number of experiments. The superimposed curve is a fit according to the Hill equation (eq. 1) yielding $I C_{50}=1.37 \pm 0.11 \mathrm{nM}$, and $n_{H}=1.14 \pm 0.07$ for F-clofilium. As a control, block by clofilium is also indicated as dashed curve (data from Gessner \& Heinemann, 2003): $I C_{50}=0.83 \mathrm{nM}$, and $n_{H}=1.01$.

Xenopus oocytes, F-clofilium blocked almost indistinguishably to clofilium: the apparent $I C_{50}$ values were $1.37 \mathrm{nM}$ for F-clofilium as compared to $0.83 \mathrm{nM}$ for clofilium (Gessner et al., 2003) (Figure 2). Thus, the extra fluoride moiety F-clofilium does not strongly impair the binding of the drug inside the EAG channel pore. Given the strong structural similarity of hEAG1 and hERG1 channels (Gessner et al., 2004), these data corroborate the notion that F-clofilium is also a potent blocker of hERG1 channels once it has passed the plasma membrane.

\section{Biodistribution of ${ }^{18} \mathrm{~F}$-clofilium in mice}

The biodistribution of F-clofilium was tested in living mice. For that purpose, ${ }^{18} \mathrm{~F}$-clofilium was injected into the tail vein of Balb/c female mice. The mice were
Table 1. Biodistribution of ${ }^{18} \mathrm{~F}$-clofilium in Balb/c female mice. The data indicate the decay-corrected relative activity (\% injected dose/g); the errors indicate SD values from four animals each.

\begin{tabular}{lcc}
\hline \multicolumn{1}{c}{ Tissue } & $\begin{array}{c}\text { Activity at } 15 \mathrm{~min} \\
(\% \mathrm{ID} / \mathrm{g})\end{array}$ & $\begin{array}{c}\text { Activity at } 60 \mathrm{~min} \\
(\% \mathrm{ID} / \mathrm{g})\end{array}$ \\
\hline Blood & $0.22 \pm 0.06$ & $0.05 \pm 0.01$ \\
Liver & $2.36 \pm 0.26$ & $1.09 \pm 0.14$ \\
Lung & $1.09 \pm 0.10$ & $0.98 \pm 0.02$ \\
Spleen & $0.97 \pm 0.19$ & $0.46 \pm 0.07$ \\
Kidney & $11.23 \pm 0.07$ & $5.36 \pm 0.45$ \\
Stomach & $0.45 \pm 0.14$ & $0.34 \pm 0.03$ \\
Femur & $0.79 \pm 0.15$ & $1.37 \pm 0.09$ \\
Muscle & $0.27 \pm 0.05$ & $0.26 \pm 0.02$ \\
Thyroid & $0.21 \pm 0.03$ & $0.21 \pm 0.02$ \\
Intestine & $2.87 \pm 0.38$ & $4.03 \pm 0.10$ \\
Brain & $0.03 \pm 0.00$ & $0.02 \pm 0.00$ \\
Heart & $2.64 \pm 0.28$ & $2.38 \pm 0.23$ \\
\hline
\end{tabular}

sacrificed at two different times after injection (15 min or $60 \mathrm{~min})$. The data are shown in Table 1.

The kidneys showed the highest uptake of radioactivity both at $15 \mathrm{~min}$ and $60 \mathrm{~min}$ when it had dropped down to half of its former value. Relatively high enrichment was found in the intestines with enhancing tendency over the time. Bones represented by the femur showed a relatively small initial uptake but some enhancement after 60 min, which might be a result of metabolism to free radioactive fluoride. This metabolism might be caused by the liver, also showing decreasing activity over time.

In the blood radioactivity had disappeared nearly completely after $60 \mathrm{~min}$. The ratio of activity in heart to blood was 12 after $15 \mathrm{~min}$ and 48 after $60 \mathrm{~min}$. A relatively high radioactivity was found in the myocardium with nearly identical values after 15 and $60 \mathrm{~min}$. Based on these initial data, we assumed that imaging of the dog myocardium by PET might be successful.

\section{Biodistribution of ${ }^{18} \mathrm{~F}$-clofilium in a living dog}

Subsequently the biodistribution of ${ }^{18} \mathrm{~F}$-clofilium in a living animal was investigated by PET scan. Given the anatomical and physiological similarities to the human heart we chose the canine model. Abundant expression of cERG messenger ribonucleic acid (mRNA), strongly related to hERG1 (Zehelein et al., 2001), led to the expectation of detecting binding of ${ }^{18} \mathrm{~F}$-clofilium to the dog myocardium using PET. Results of this investigation are shown in Figure 3.

Strong enrichment of radioactivity was seen only in the kidneys, the bladder and the bones (Figure $3 \mathrm{~A}$ ). The radioactivity in bones of the dog, as also found in the femur of mice (s. Table 1), is most likely due to an 

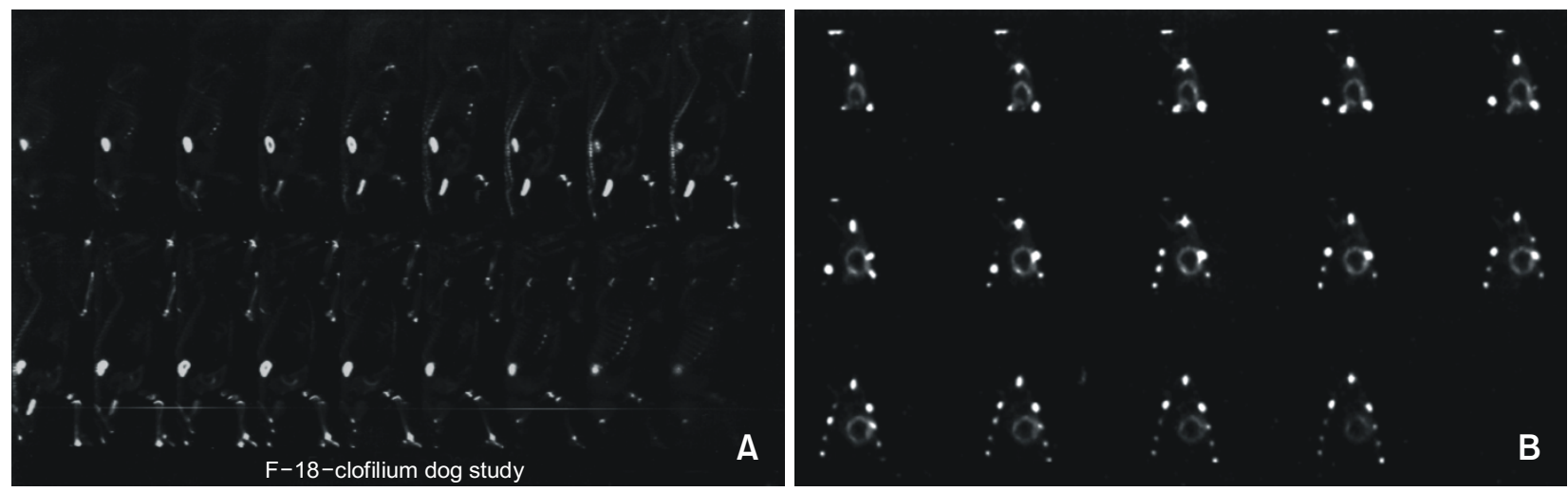

Figure 3. (A) ${ }^{18} \mathrm{~F}$-clofilium PET scan of a dog ( $25 \mathrm{~kg}$, male, beagle). Shown is a series of whole-body images as coronal (horizontal) slices of a dog positioned on its left side. High uptake of radioactivity is found in the kidneys, bladder and bone. The first slices show a kidney, the more central slices a kidney and the bladder, then followed by the second kidney. Slice thickness $10 \mathrm{~mm}$. (B) Series of sagittal PET scan slices (vertical, thickness $5 \mathrm{~mm}$ ) of the thorax of the dog positioned on his left side. High enrichment of radioactivity is found in the ribs, minor activity is found in the myocardium depicted as red circles cut vertical to the short axis of the heart.

uptake of ${ }^{18} \mathrm{~F}$-fluoride, which is a strong bone-seeking ion (Hoh et al., 1993). Therefore also some elimination of fluoride must have taken place (Degrado et al., 1992).

Compared to the biodistribution in mice, enrichment of radioactivity in the kidneys of the dog seemed to be more dominant.

The strong uptake by the kidneys is certainly not due to free ${ }^{18} \mathrm{~F}$-ions, since enrichment of ${ }^{18} \mathrm{~F}$-ions in this amount in organs such as kidneys is not observed (Hoh et al., 1993). Thus, we can assume a strong binding of the ${ }^{18} \mathrm{~F}$-clofilium cation or perhaps of a radioactive metabolite to the kidneys and subsequently a secretion into urine collected in the bladder. The cause of this very strong binding of activity to the kidneys could be due to a high density of high-affinity binding sites in the kidney tissue. These binding sites should be distinct from cERG1 $\mathrm{K}^{+}$channels, since such channels are not strongly expressed in the kidney (Zehelein et al., 2001).

Despite the strong uptake and the fast clearance rates of the radioactivity by the kidneys, metabolization to ${ }^{18} \mathrm{~F}$-ions and their uptake by bones, it was possible to depict the myocardium by PET as shown in Figure 3B. The myocardium is shown in form of circles cut vertical to the short axis of the heart.

Neighboring bones (rips, breastbone) are disturbing the myocardial imaging by the "partial volume effect" (Hatazawa et al., 1991), caused by the limited resolution $(5 \mathrm{~mm})$ of the method. This results in an overestimation of radioactivity in parts of the myocardium in the neighborhood of bones taking up high amounts of ${ }^{18} \mathrm{~F}$-ions.

Thinning of the myocardium in the region of the atria results by partial volume effect in an underestimation of the activity in the atrial walls. With this in mind we conclude that F-clofilium binding sites are nearly homogeneously distributed throughout the myocardium.

Recently, Angelo et al. reported an even distribution of the radioiodinated scorpion toxin $\left[{ }^{125} \mathrm{~J}\right]-B e K m-1$ in an excised rat heart and concluded a homogeneous expression of hERG channels throughout the heart (Angelo et al., 2003). F-clofilium binds as described above not only to hERG1 channels but also in low nanomolar range to hEAG1 channels. The latter have not been detected in the myocardium but only in the brain and tumor cells. Abundant expression of $\mathrm{CERG}$ mRNA in dog's heart was detected (Zehelein et al., 2001). Since cERG and hERG channel proteins are identical in their membrane-spanning segments and the pore forming region (Zehelein et al., 2001), harboring the binding site for clofilium, we conclude that the myocardial uptake of radioactivity after injection of ${ }^{18} \mathrm{~F}$-clofilium primarily reflects a binding to the cERG1 $\mathrm{K}^{+}$channels. These binding sites seem to be nearly homogenously distributed throughout the heart and our results are in support of previous result using a different method (Angelo et al., 2003).

Application of ${ }^{18} \mathrm{~F}$-clofilium by PET is able to depict the dog's myocardium probably by binding to $\mathrm{CERG}$ channels. These channels seem to be almost homogeneously distributed throughout the myocardium as far depicted. High renal uptake and fast catabolization of ${ }^{18} \mathrm{~F}$-clofilium to radioactive fluoride might compromise its application in humans for PET-scan investigations.

\section{Acknowledgement}

We are grateful for technical assistance by A. Roßner. This work was supported by the Deutsche Forschungs- 
gemeinschaft, DFG (He 2993/6), Nuclear R \& D Program from the Ministry of Science \& Technology in Korea and Dongguk University Research Fund.

\section{References}

Angelo K, Korolkova YY. A radiolabeled peptide ligand of the hERG channel [125 J]-BeKm-1. Pflügers Arch 2003;447: 55-63

Borzak S, Al-Khaled N, Douthat L, Steinberg M, Goldberg $A D$. Intraveneous clofilium for conversation of atrial fibrillation. Am J Cardiol 1997;79:94-7

DeGrado TR, Moka DC. Non-beta-oxidizable omega- $\left[{ }^{18} \mathrm{~F}\right]$ fluoro long chain fatty acid analogs show cytochrome P-450 mediated defuorination: implications for the design of PET tracers of myocardial fatty acid utilization. Int J Rad Appl Instrum B 1992;19:389-97

Gavrilova-Ruch O, Schönherr K, Gessner G, Schönherr R, Klapperstück T, Wohlrab W, Heinemann SH. Effects of imipramine on ion channels and proliferation of IGR1 melanoma cells. J Membrane Biol 2002;188:137-49

Gessner G, Heinemann SH. Inhibition of hEAG1 and hERG1 potassium channels by clofilium and its tertiary analogue LY97241. Br J Pharmacol 2003;138:161-71

Gessner G, Zacharias M, Bechstedt S, Schönherr R, Heinemann SH. Molecular determinants for high-affinity block of human EAG potassium channels by antiarrhythmic agents. Mol Pharmacol 2004;65:1120-9

Hatazawa J, Hatano K. Measurements of D2 Dopamine Receptor-Specific Carbon-11-YM-09151-2 Binding in the canine brain by PET: Importance of Partial Volume Correction. J Nucl Med 1991;32:713-8

Hoh CK, Hawkin. Whole body skeletal imaging with $\left[{ }^{18} \mathrm{~F}\right]-$ fluoride ion and PET. J Comput Assist Tomogr 1993;17: 34-41

Molly BB, Steinberg MI. Improvements in or relating to imaging agents. European Patern Publication No. 0107452A1, 14.10. 83. 1983
Pardo LA, del Camino D, Sanchez A, Alves F, Bruggemann A, Beckh S, Stuhmer W. Oncogenic potential of EAG K+ channels. EMBO J 1999;18:5540-7

Reivich M, Alavi A, Wolf A, Fowler J. Glucose metabolic rate kinetic model parameter determination in humans: the lumped constants and rate constants for $\left[{ }^{18} \mathrm{~F}\right]$ fluorodeoxyglucose and $\left[{ }^{11} \mathrm{C}\right]$ deoxyglucose. J Cereb Blood Flow Metab 1985;5:179-92

Schönherr R, Heinemann SH. Molecular determinants for activation and human inward rectifier potassium channel. J Physiol 1996;493:635-42

Schönherr R, Löber K, Heinemann SH. Inhibition of human ether à go-go potassium channels by $\mathrm{Ca}^{2+} /$ calmodulin. EMBO J 2000;19:3263-71

Schönherr R, Rosati B, Hehl S, Rao VG, Arcangeli A, Olivotto M, Heinemann SH, Wanke E. Functional role of the slow activation properties of ERG $\mathrm{K}^{+}$channels. Eur $\mathrm{J}$ Neurosci 1999;11:753-60

Schwaiger M, Rodney $\mathrm{H}$. The Clinical Role of Metabolic Imaging oftheHeart by Positron-Emission-Tomography. J Nucl Med 1991;32:565-78

Su KSE, Campanale KS, Gries CL. Nasal drug delivery system of a quaternary compound: clofilium tosylate. J Pharm Sci $1984 ; 73: 1251-4$

Suessbrich H, Schönherr R, Heinemann SH, Lang F, Busch AE. Specific block of cloned Herg channels by clofilium and its tertiary analog LY97241. FEBS Lett 1997;414:435-8

Vandenberg JI, Walker BD, Campbell TJ. HERG $\mathrm{K}^{+}$channels: friend and foe. Trends Pharmacol Sci 2001;22:240-6

Wang H, Zhang Y, Cao L, Han H, Wang J, Yang B, Nattel S, Wang Z. HERG $\mathrm{K}^{+}$channel, a regulator of tumor cell apoptosis and proliferation. Cancer Res 2002;62:4843-8

Yu KH, Kim YS, Kim SW, Park JH, Yang SD, Herdering W, Knoechel A. Synthesis of $\left[{ }^{18} \mathrm{~F}\right]$ Fluoroclofilium as a potential cardiac imaging agent for PET Studies. J Label Compd Radiopharm 2003;46:1151-60

Zehelein J, Zhang W, Koenen M, Graf M, Heinemann SH, Katus HA. Molecular cloning and Expression af cERG, the ether a go-go-related gene from canine myocardium. Pflügers Arch 2001;442:188-91 